\title{
Novel Porcine Models of Myocardial Ischemia/Infarction - Technical Progress, Modified Electrocardiograms Validating, and Future Application
}

\author{
Jianxun Liu and Xinzhi Li \\ Department of Pharmacology, Xiyuan Hospital, \\ China Academy of Chinese Medical Sciences, Beijing
}

China

\section{Introduction}

Cardiovascular diseases, the first killer for human being, constitute the global economic burden. The updated data from World Health Organization (WHO) addresses that the mortality rank of myocardial infarction will increase from No.5 in 2000 to Top One till 2020. And 30 percent of overall mortality is due to the cardiovascular diseases. Their prevalence has severely affected patients' healthy conditions. Therefore, it is the priority for us to enforce the research on the mechanisms of pathogenesis and treatment of cardiovascular diseases.

Heart attacks are most commonly caused by a fatty "plaque" dislodging from the blood vessel wall. Inflammation in vessels and heart also plays an important role in this process. This provokes tiny cells in the bloodstream known as platelets to clump together and block the blood circulation to important organs. To elucidate the exact mechanism of the mentioned process, researchers need to develop various pre-clinical approaches to simulate the clinical presentation in animal models, cell strains, or even in tube reactions. Among those methodologies, animal model are more acceptable than the others due to its easily "from bench to bed" translational property. The use of appropriate large-animal models is essential if some new therapeutic strategies are to be critically evaluated in a preclinical setting before their use in humans.

Over the past decade, swine have been increasingly used in studies of myocardial ischemia because of their numerous similarities to humans, including minimal preexisting coronary collaterals as well as similar coronary anatomy and physiology. In this chapter, the author will consequently review the most commonly used swine models of myocardial ischemia with special attention to regional myocardial blood flow and function and critically evaluates the strengths and weaknesses of each model in terms of utility for preclinical trials.

Also in this part, several porcine models of myocardial ischemia/infarction developed in our department will be summarized briefly regarding with the technical progress. We would also like to share our experiences about new adventures in the field of electrocardiogram mapping using multiple electrodes. 


\section{Animal models of myocardial ischemia/infarction}

\subsection{Myocardial ischemic or infarction models}

High mortality and morbidity associated with myocardial ischemia or even the infarction necessitates modelling the process in animal. In a hospital setting, because the primary concern is resuscitation and maintaining of the patient's life, but not research in the underlying mechanism of the diseases. To study the pathophysiological changes associated with myocardial ischemic injury and how they lead to the establishment of cardiac cell death and regional infarction, animal models of clinical pathophysiology should gain our serious concern to overcome the limitations of clinical studies and to play an invaluable role in advancing mechanistic insight. Animal model studies will considerably provided new ideas and hypotheses that have been tested in clinical studies and have thus increased the level of knowledge regarding cardioprotection in the ischemic milieu.

Moreover, the use of experimental models of heart diseases in animals is an obligatory step for the understanding of mechanisms involved in pathologies consecutive to cardiac metabolic or functional disorders. As for any kind of therapeutic approach, to demonstrate its efficacy and safety there is also a need for animal models. Among factors of importance, there are animal species, coronary thrombosis induction method, thrombus composition and site of formation.

\subsubsection{Acute ischemic and reperfusion animal models}

Based on the clinical profiles of the sudden heart attach, people firstly sought for the mimic method to study myocardial ischemic condition by ligation of one of the three main arteries in heart. Back to 1975, myocardial ischemia was induced in swine by ligation of the anterior descending coronary artery for periods of 30 minutes to six hours. Electron microscopy was used to monitor normal and ischemic myocardium (Lichtig et al., 1975). Scientists from our institute did contribute quite a lot to this model, not in swine, but in dogs. The dog's heart was exposed by opening the chest. The acute ischemia models were prepared by ligating left anterior descending (LAD) artery. Meanwhile, timed monitoring hemodynamics and blood flow were determined by physiological polygraph and electromagnetic flow meter, respectively $(\mathrm{Li}, 1978)$. In another research group, the diagonal branches of the left anterior descending coronary artery in pigs were ligated for 5, 15, 30, 60 and 120 minutes. By using transmission electron microscopy, morphological observations indicated that initial leukocyte infiltration and inflammation during myocardial ischemia (Park et al., 1985).

These kinds of open-chest model were widely accepted during 1990s and were still prevalent in 2000s. With development of the diagnostic methods, more and more advanced technologies were employed in the research of heart diseases and animal modelling. In one of representative works, nine open-chest swine undergoing myocardial ischemia were instrumented for measurement of regional myocardial blood flow (microsphere method), contractile function (sonomicrometry), and hemodynamics. L-[1-14C] Lactate or L-[U-13C] lactate was infused intravenously using a primed continuous infusion technique to quantify regional myocardial lactate release. A close inverse relation between regional myocardial lactate release and regional subendocardial blood flow during graded ischemia was revealed (Guth et al., 1990). When researcher evaluated the utility of transplanting bone marrow stromal cells in a porcine myocardial infarction model, a myocardial infarction was created by occluding the distal left anterior descending artery in pigs with coils and Gelfoam sponge (Tomita et al., 2002). In this study, sestamibi technetium single-photon 
emission computed tomographic scans were performed to compare the stroke volume, regional perfusion, and wall motion in different treatment group.

Most of these myocardial infarction animal models using dogs or pigs is created by coronary artery ligation after surgical opening of the chest and exposure of the heart. Hereby, numerous disadvantages and experimental limitations may be associated with this technique: There is evidence suggesting that normal cardiac mechanics are disturbed in acute models after thoracotomy. Opening the chest and pericardium have both been suspected and reported to influence the pattern of left ventricular remodeling in chronic models (Kraitchman et al., 2000; Ludemann et al., 2007). Furthermore, the surgical trauma may cause a high rate of complications, resulting in high mortality. In addition, the trauma may render successful recovery from anesthesia in models of myocardial infarction more difficult, compared with closed chest procedures.

Besides, to date, there are various myocardial ischemic animal models, such as ligating the coronary artery (Takahashi et al., 2005), placing constrictors on the coronary artery (Laham et al., 2000), putting microembolus into the coronary artery (Huang et al., 2004), or injecting ferric chloride via the vein (Dogne et al., 2005). However, operations in these methods lead to much damage in animals, and channelization can not be restored in the obstructed coronary artery. More considerably, the mimic pathophysiological alterations deviate from clinical data, especially autopsy. Nowadays, cardiovascular medicine research requires the availability of appropriate experimental animal models that are as close to humans as feasible.

\subsubsection{Mechanically chronic ischemic animal models}

The most widely used porcine model of chronic ischemia has been the ameroid constrictor. Originally described by Litvak and colleagues in 1957, these constrictors are constructed of the hygroscopic material casein encased within a steel sleeve. When the device is implanted around an artery, the constrictor absorbs water and swells, compressing the artery and producing total coronary occlusion over a period of 14-30 or more days (Elzinga, 1969). The major advantage of the ameroid constrictor model is its simplicity. Inherent limitations to the use of these occluders include an inability to control the rate or degree (sometimes incomplete) of coronary occlusion (Inou et al., 1980).

Another large-animal model of chronic ischemia has involved placing an adjustable hydraulic occluder around an epicardial coronary artery to produce a fixed degree of coronary stenosis (Bolukoglu et al., 1992). This model is similar to the fixed stenosis model in that the coronary artery is reduced in diameter by an external device, although with this latter model the occluder is typically placed either proximal or distal to a myocardial flow probe. Another famous study using this animal is about the relationship between hibernating myocardium and cell autophage (Yan et al., 2005). Chronically instrumented pigs were studied with repetitive myocardial ischemia produced by coronary hydraulic stenosis. Autophagy, triggered by ischemia, could be a homeostatic mechanism, by which apoptosis is inhibited and the deleterious effects of chronic ischemia are limited (Yan et al., 2006).

\subsubsection{Swine models with thrombosis formation in coronary artery}

Coronary artery thrombosis is widely accepted as a major cause of myocardial infarction (MI). The abrupt transition from a stable, often clinically silent, disease to a symptomatic 
life-threatening condition results from endothelial injury or plaque disruption followed by thrombosis (Falk et al., 1995). Because of this acute intracoronary thrombotic occlusion, the primary goal of therapy is rapid, complete, and sustained restoration of infarct-related artery blood flow. In this case, the choice of the animal model of thrombosis to evaluate the efficiency of antiplatelet, antithrombotic or thrombolytic drugs in preclinical studies is crucial. Numerous animal models of thrombosis-induced MI have been proposed in the last decades (Leadley et al., 2000). Another example was from porcine model of myocardial infarction induced by topical application of ferric chloride to the LAD coronary artery, which was validated to quite close to clinical pathophysiological conditions, such as thrombus formation occurring after atherosclerotic plaque rupture (Dogne et al., 2005).

In dogs, left circumflex coronary artery (LCx) thrombosis was induced by vascular electrolytic injury (Hennan et al., 2001; Romson et al., 1980). After a flow probe and stenosis had been placed around the LCx, an intracoronary electrode was inserted through the LCx arterial wall, with the uninsulated portion positioned against the endothelial surface. An anodal current of $150 \mu \mathrm{A}$ was applied to the endothelial surface to initiate the experiment. This operation is relatively difficult to master or needs much more practice, since the electrode is introduced into coronary artery. Another shortcoming is bleeding due to artery injury during procedure, especially the introduction of electrode.

In our department, to evaluate the thrombolytic effects of heparin-like drugs, swine's endoarterium was injuried and coronary thrombi were formed gradually through direct electrical stimulation on the coronary artery of animals (Liu et al., 2002). However, some limitations of this model should be pointed out. First, we are aware of the fact that the maintenance of pigs is expensive and difficult, and requires special facilities that are beyond the capabilities of most laboratories. Secondly, the pig presents a high myocardial sensitivity to hypoxia. Acute reduction of coronary blood flow can easily induce paroxystic ventricular fibrillation which may then requires the use of prophylactic antiarrythmic agents.

\subsubsection{Making myocardial ischemic animal models via interventional techniques}

Most models listed above in the pig, with or without subsequent reperfusion need thoracotomy. The majority of these employ surgical techniques of coronary occlusion following thoracotomy. During these complex operations, the subtle enviorment inside of the chest will be somewhat disturbed. The endovascular and minimal invasion techniques offer a series of advantages over the surgical technique such as a lower incidence of infections and of related complications which can compromise the survival of the animal and the validity of the results.

These disadvantages raise the demand for closed-chest models for myocardial infarction in large animals. To facilitate the surgical operation, we introduced the intervention technique and selective coronary angiography into our study. One ischemic model we used was developed in Chinese miniature swine, where myocardial ischemia was performed by injecting self-embolus into the middle segment of the left anterior descending (LAD) without thoracotomy (Liu et al., 2007b; Yu et al., 2007b). Embolization occurred in the LAD coronary artery of the Chinese miniature swine injected by self-embolus. There were significant myocardial ischemia and large cardiac muscle infarction in the Chinese miniature swine, which were accompanied with increased Body surface ECG (BS-ECG) (Liu et al., 2007b), decreased hemodynamic indexes of the cardiac output, cardiac index, left cardiac work and left cardiac work index, and increased systemic vascular resistance index. 
Pathohistological analysis revealed myocardial degeneration, necrosis, fibrosis, inflammatory cell infiltration and granulation tissue hyperblastosis. Our data showed that myocardial ischemia induced by injecting self-embolus into the LAD coronary artery in Chinese miniature swine is quite close to clinical pathophysiological conditions. (Fig. 1.)
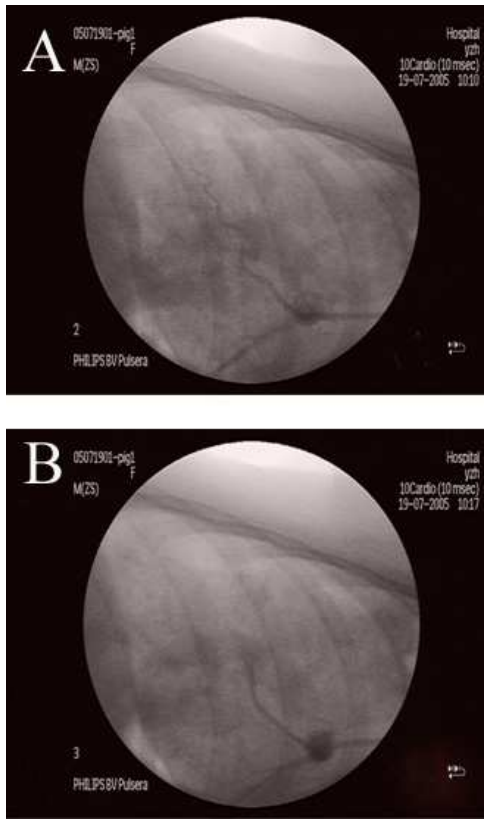

Fig. 1. Changes of coronary embolism were disclosed by the coronary angiography. (A) the LAD of the model group was normal at pre-operation; (B) the LAD of the model group was completely and instantly embolized, post-operation.

In other lab, injection of thrombogenic material, such as microcoils or thrombin fibrinogen mixtures, have also been proposed and explored (Koning et al., 1993; Naslund et al., 1992). In these studies, after injection into a coronary artery, the blood flow transports these materials downstream until their size matches the diameter of the coronary artery. Standardization of the infarction size is not possible because there is physiologic variation of the diameter of the coronary arteries between individual animals of similar body weight. Furthermore, with these approaches, either reperfusion is not possible or occurs after spontaneous lysis of the occluding material. This approach does not allow for controlling the duration of the occlusion.

On the other hand, X-ray-guided placement of a balloon catheter in the coronary artery and inflation of the balloon for coronary artery occlusion avoids extensive surgery and allows the control of both the location and the time of occlusion. This approach has been used in several studies. Balloon occlusion of the LAD can successfully be performed in pigs to create reperfused or occlusive myocardial infarction. The technique allows for a relatively short preparation time compared with open-chest surgery, control over the duration of ischemia, and standardization the location of the occlusion and infarcts size. The resulting changes of the myocardial function are solely due to the ischemic injury. This renders results obtained 
with this model more easily interpretable. Furhtermore, this model is advantageous for MRI studies because the heart is not exposed, so that susceptibility artifacts that arise from the myocardium-air border in open chest-models are avoided (Krombach et al., 2005).

\subsubsection{Coronary atherosclerosis in swine}

Recently, advanced coronary atherosclerosis in swine was produced by combination of balloon-catheter injury and cholesterol feeding (Li et al., 2009). In this study, twelve Chinese experimental miniature swine (CEMS) were randomly divided into Control and Model groups. In the model, fed with high fat diet for 2 weeks, the pigs underwent left coronary angiography and balloon over dilation in left anterior descending artery (LAD) followed by continuous feeding with high fat diet for 8 weeks. At the end of 10 weeks, an IVUS catheter was guided to the LAD artery. Intravascular ultrasound and virtual histology (IVUS-VH) data analysis was based on border contour calculation from gray scale. Coronary angiography with (IVUS) and virtual histology (VH) will help us to detect the process of coronary atherosclerosis during coronary heart disease (CHD). IVUS classified the plaque as concentric, with fibrous $62.8 \%$, fibro-lipidic $12.7 \%$, necrotic $15.5 \%$, and calcific $8.9 \%$ tissue. In vitro histopathology correctly identified the different presence of fibrous tissue, and different extent of stenosis, which was consistently correlative to the result of IVUS-VH (Fig. 2.). These models provide essential help for researchers to better understand the pathophysiological implications of the heart diseases, from endothelium injury to plaque stability, from marker protein expressions to cell death pattern. All these findings will not only present one of the scientific answers to the pathogenesis of model animals with coronary atherosclerotic heart diseases (coronary heart disease, $\mathrm{CHD}$ ), but also will facilitate to interpret the scientific insights of prevention and therapeutic strategy.

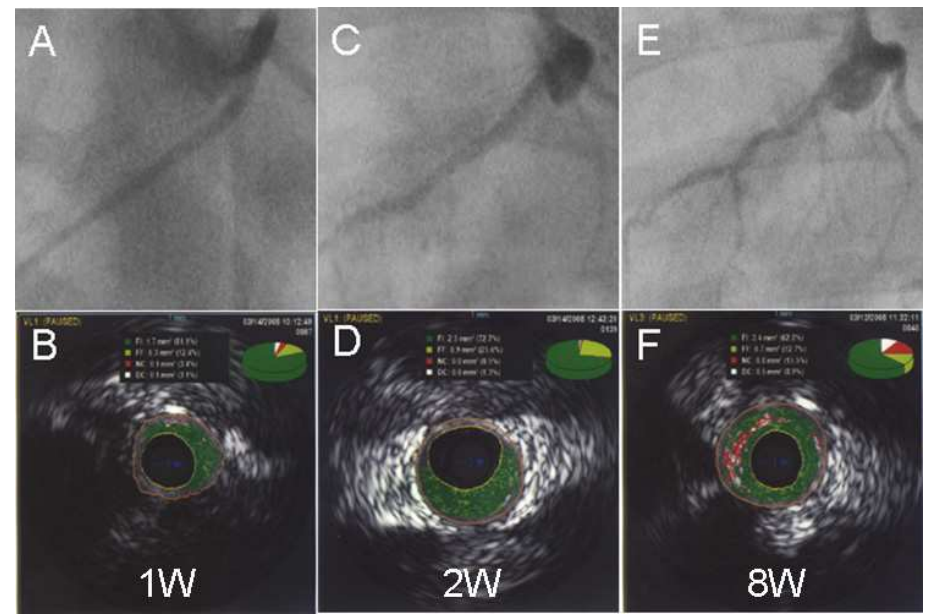

Fig. 2. LAD angiography and IVUS-VH after balloon injury in swine fed with high-fat diet for different time $(1,2,8 \mathrm{w})$. (A, C, E) LAD angiography; (B, D, F) IVUS-VH.

\subsubsection{Transgenic animal models for cardiovascular diseases}

For economic reasons and with the help of genetically targeted mouse manipulated in the laboratory, considerable basic and applied cardiovascular experimentation has been 
performed in mice and rats; on the assumption that the results can be translated to humans. Transgenic mice are widely accepted and used in many labs. To delve the roles of prostaglandin $\mathrm{H}$ synthase- 1 and prostaglandin $\mathrm{H}$-synthase-2, known colloquially as COX-1 (target of aspirin: first level heart disease prevention drug) and COX-2 (target of many antiinflammatory drugs), in cardiovascular systems, Four different strains of mice were successfully generated and characterized including: (i) genetic knockdown of COX-1 (8097\% reduction) (Yu et al., 2005); (ii) genetic knockdown of COX-2 (80-95 \% reduction) (Seta et al., 2009); (iii) knock-in of a point mutation at the COX-2 active site to abolish cyclooxygenase activity and leave peroxidase activity intact Ptgs2Y385F (Yu et al., 2006); and (iv) exchange of COX-1 into the COX-2 locus (Yu et al., 2007a). But before we head to set up more transgenic knock-in and/or knock-out mice, we should keep our own brain cool to think over the necessity and specific characters in each animal model.

Tiny mouse's heart muscle mass $(<100 \mathrm{mg})$ as compared to about $500 \mathrm{~g}$ in the human heart presents problems of magnitude and architectural complexity. This may partially explain why so many effective investigational new drugs withdraw market after phase 3 clinical trials even they have show promising pharmacological effects in experimental animal models. Scientist resorted to another popular rodent animal: rat. But hampered by lacking specific techniques to manipulate the rat stem cells which have been working very well in mice, rats were kicked out of the transgenic game for a long time. Zinc-Finger Nucleases (ZFN) technology to generate knock-in rats in which foreign genes have been inserted, or 'knocked-in', into the rat genome in a precisely targeted manner (Geurts et al., 2009). This breakthrough achievement represents a major step forward in the creation of a transgenic animal, which may serve as more predictive models of human disease, especially in cardiovascular diseases. For example, first rat knockout in the renin-angiotensin system has demonstrated the efficacy of the ZFN technology for creating knockout rats for cardiovascular disease on any genetic background (Moreno et al., 2011).

\subsubsection{Computer-based simulation of ischemia and reperfusion model}

Experimental models, however, have their own set of limitations that hamper the comprehensive evaluation of heart functional mechanisms. At the end of the last century, some scientists pursued to develop a biochemically and biophysically detailed model that could provide a novel approach to studying myocardial ischaemia and reperfusion (Ch'en et al., 1998). Over the last decade, analysis of electrophysiological phenomena following coronary occlusion has been significantly augmented and advanced by the use of mathematical modeling and computer simulations, from the ionic channel to the whole organ. One of the major contributions of computational research in electrophysiology has been the ability of mathematical models to dissect various effects and to tease out important relations between electrophysiological parameters. This part has been well reviewed by others (Rodriguez et al., 2006).

\subsection{Evaluation of myocardial ischemia and infarction}

Conventional 12-lead electrocardiography (ECG) as well as analysis of serum markers still play a non-replaceable role clinically and even in the animal model research. In addition, a number of special techniques such as echocardiography, nuclear magnetic resonance imaging (MRI) (Wright et al., 2009), computed tomography (CT) (Baks et al., 2006; Buecker et al., 2005; Mahnken et al., 2005), single photon emission computed tomography (SPECT), 
and positron emission tomography (PET) have been devised to support diagnosis in the patients who show ambiguous symptoms and ECG findings. Major advantage of these latter methods is that infarct size can be non-invasively and repeatedly measured in vivo. Major limitation of the non-invasive imaging techniques in humans is the lack of quantification of the risk zone.

In preclinical small animal models, the use of above mentioned novel non-invasive imaging techniques is also limited due to several technical problems (e.g. lack of area at risk determination, insufficient temporal and spatial resolution, irradiation, etc.) and their high cost. Therefore, direct post-sacrifice techniques such as e.g. the triphenyltetrazolium (TTC) staining as well as nitroblue tetrazolium (NBT) staining, is still the most widely used, lowcost method to assess infarct size in animal models.

\subsubsection{Modified ECG and multiple-electrode mapping}

Identifying patients at risk of ST segment elevations (most frequently observed in heart attack) by use of body surface electrical measures is controversial. To probe the principal variations during myocardial ischemia, scientists have pursued to measure the electrophysiological changes of heart by modified ECG (s). In our lab, to circumvent some of these problems and to further our knowledge of the cardiovascular disorders we have undertook a series of investigations that consisted of designing novel modified ECG since 1970s. Two different modified ECGs were successfully developed and characterized including: epicardial electrograms (Li, 1978; Liu et al., 2002) and body surface ECG (BSECG) (Liu et al., 2007b). See the section "EECG and BS-ECG" below for the details.

Other lab worldwide besides our lab should also be reviewed here as to design very sensitive, multiple-electrode mapping technology. Mimicking the body 12-lead ECG, scientists from University of Oxford recorded ventricular epicardial electrograms from 5 anesthetized pigs with a 127-electrode sock and simultaneously investigated torso ECG using a specifically designed vest with 256 ECG electrodes. One of breakthroughs they have made in this study is that with chest reclosed, simultaneous arrays of epicardial electrograms and torso ECGs can be recorded during LAD occlusion and reperfusion (Nash et al., 2003).

The earlier identification of coronary occlusion with ST-segment elevation, the better outcomes patients undergoing heart attack may have due to timing of revascularization. Using an implantable, high-fidelity, intracardiac electrogram monitoring system with longrange telemetry in porcine subjected to acute coronary occlusion precipitated by stent thrombosis, researchers from Michigan State University real-timely detected acute STsegment elevation and analyzed its correlations with thrombotic coronary occlusion. High sensitivity and specificity (100\% and 100\%, respectively) in such a system make it possible to advance the time frame of reperfusion therapy and potentially prevent, rather than interrupt, acute myocardial infarction in patients with coronary artery disease (Fischell et al., 2006).

In a porcine acute infarct and reperfusion model induced by balloon occlusion of the left anterior descending coronary artery for 45 minutes, endocardial electromechanical mapping (EMM) was performed to evaluat the extent of myocardial ischemia. Even though there was significant intersegmental threshold variability at baseline and after infarction, the electromechanical activity thresholds, for infarct detection, could be established. In this study, the capacity of separating myocardium with evolving necrosis from viable 
myocardium was so promising that would result in potential clinical uses (Odenstedt et al., 2003).

\subsubsection{Advanced imaging systems}

Conventional methods to quantify infarct size after myocardial infarction in mice are not ideal. Cardiologists therefore implemented a fast, high-resolution method to directly measure infarct size in vivo using three-dimensional (3D) late gadolinium enhancement MRI (3D-LGE). They had validated an improved 3D MRI method to noninvasively quantify infarct size in mice with unsurpassed spatial resolution and tissue contrast. This method is particularly suited to studies requiring early quantification of initial infarct size, for example, to measure damage before intervention with stem cells (Bohl et al., 2009). Another study group combined the small-animal PET and MRI data to acquire quantitative in vivo insights into cardiac pathophysiology, and sought to determine the feasibility of PET and MRI for the quantification of ischemic injury in the rat model. Successful integrating information from small-animal PET and clinical MRI instrumentation allows for the quantitative assessment of cardiac function and infarct size in the rat model. The MRI measurements of scar can be complemented by metabolic imaging, addressing the extent and severity of ischemic injury and providing endpoints for therapeutic interventions (Higuchi et al., 2007).

\subsubsection{Histological analysis of infarct size}

In preclinical studies, still the postmortem histological analysis is considered to be the gold standard for measuring infarct size. However, there are a number of disadvantages of this technique, making a reliable non-invasive alternative highly desirable. First, histological methods leave no residual tissue for further analysis. Second, visual interpretation and planimetry of heart sections may be subjective in cases with poor viable/non-viable contrast due to hemoglobin residues within the necrotic regions or with TTC-induced geometric distortion of the sample. Finally, animals must be euthanized to measure injury, meaning longitudinal studies require separate groups of animals for each time point (Bohl et al., 2009).

Conventional TTC staining allows the quantification of infarct size much sooner than standard histological techniques, and has been shown to be equally sensitive and specific. Therefore, the direct post-sacrifice techniques such as e.g. the TTC staining, is still the most widely used, low-cost and high throughput method to assess infarct size in animal models (Skrzypiec-Spring et al., 2007).

\subsubsection{Other novel methodologies}

Many new methods were employed in cardiovascular functional measurement after ischemia or infarction. By using a three-axis accelerometer, researchers developed a novel technique for continuous real-time assessment of myocardial ischaemia in 14 anaesthetized open-chest pigs. Two accelerometers sutured on the left ventricle (LV) surface in the perfusion areas of the left anterior descending (LAD) and circumflex (CX) arteries, measured acceleration in the longitudinal, circumferential, and radial directions, and the corresponding epicardial velocities were calculated. The accelerometer had the ability to distinguish ischaemia from interventions altering global myocardial function, by which the myocardial ischaemia can be monitored in a continuous real-time mode (Halvorsen et al., 
2009). With the support of interventional techniques, intravascular ultrasound probe or intravascular Doppler velocimetry can be introduced into coronary artery and epicardial cross-sectional area and coronary flow velocity can be detected (Hutchison et al., 2005). Some study indicated that endocardial voltage amplitudes were closely related with sustaining myocardial ischemia or infarction. LV endocardial unipolar voltage (UpV) mapping was performed using the Biosense 3D navigation system 4 weeks after ameroid constrictor placement around the left circumflex coronary artery. Meanwhile, echocardiography was used to assess regional changes in myocardial wall thickening (MT) and fluorescent microspheres (4 $\times 10$ /injection) were used to quantify rest regional myocardial blood flow (MBF) in ischemic (left circumflex) and remote non-ischemic (left anterior descending) regions (Fuchs and Kornowski, 2005).

\subsection{EECG and BS-ECG}

Once a new myocardial ischemic/infarction model was to be established, validating it should be placed on the first priority. Without any doubt, traditional electrocardiogram is of first choice. Since 1970 last century, we have made much effort in developing practically modified electrocardiograms. The first one is so-called 30-point epicardial electrocardiogram (EECG), which was introduced in exposed heart. The multi-point epicardial electrodes were sutured on the ventricular surface and physiological monitor was connected to record the electrocardiogram (EECG). (Fig. 3. ) ST segment elevated more than $2 \mathrm{mV}$ was regarded as ischemic criterion to calculate the degree of myocardial ischemia (total $\mathrm{mV}$ of ST segment elevating, $\Sigma-\mathrm{ST}$ ) and myocardial ischemic scope (total point number of ST segment elevating, N-ST) (Liu et al., 2007a; Liu et al., 2002; Yu et al., 2007b). (Fig. 4. and 5.)

A

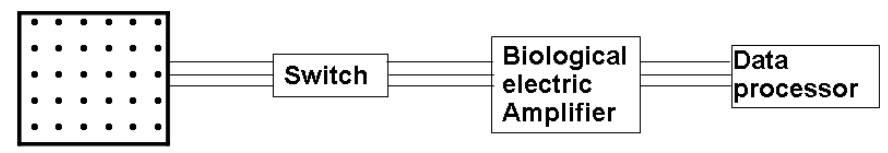

30-point electrode

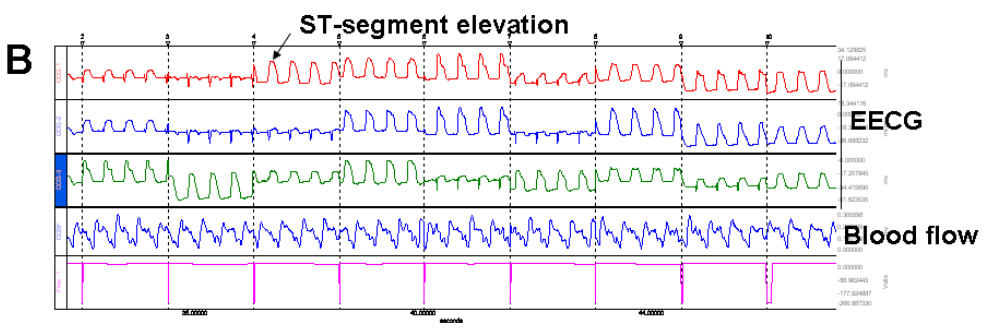

Fig. 3. Schematic components of EECG mapping system (A) and representative EECG in swine (B).

Body surface electrocardiogram (BS-ECG) is another well designed method, especially for closed chest and repeated measurement during a long period. The 30 point electrode was placed on the chest surface in the cardiac projective area and then connected to recorder 


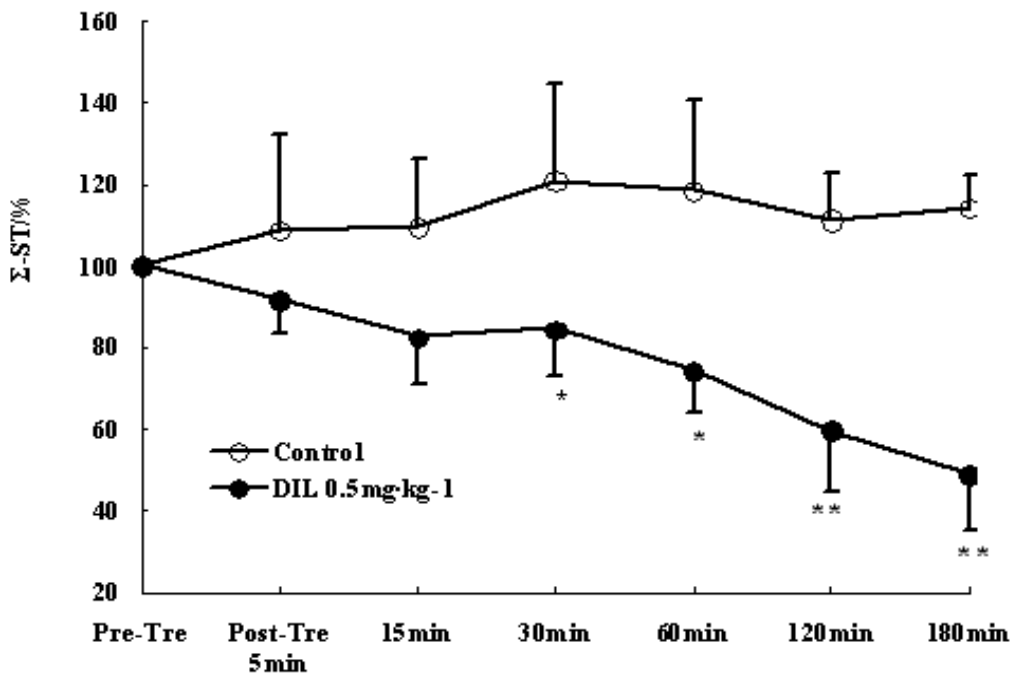

Fig. 4. The degree of myocardial ischemia ( $\Sigma$-ST) determined by the epicardiogram mapping. We proposed that the percent of ischemic condition (before treatment) was $100 \%, \Sigma$-ST \% was obtained by the comparison with ischmemic condition. DIL, diltiazem, a classical calcium channel blocker. Pre-Tre: pre-treatment, Post-Tre: post-treatment. ${ }^{*} \mathrm{P}<0.05$, ${ }^{* *} \mathrm{P}<0.01$ vs Control.

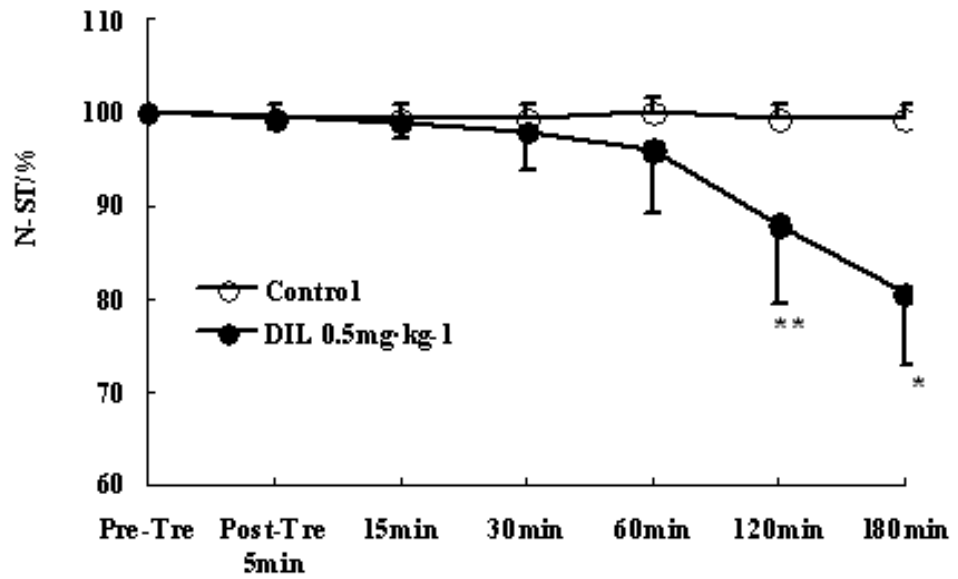

Fig. 5. The scope of myocardial ischemia (N-ST) determined by the epicardiogram mapping. We proposed that the percent of ischemic condition (before treatment) was $100 \%$, N-ST \% was obtained by the comparison with ischmemic condition. DIL, diltiazem, a classical calcium channel blocker. Pre-Tre: pre-treatment, Post-Tre: post-treatment. ${ }^{*} \mathrm{P}<0.05,{ }^{* *} \mathrm{P}<0.01$ vs Control. 
(Fig. 6.) (Liu et al., 2007b). Differently from EECG criteria due to relatively lower voltage on the skin, in BS-ECG, ST segment elevated to more than $0.8 \mathrm{mV}$ was regarded as criterion to calculate the degree of myocardial ischemia (total $\mathrm{mV}$ of ST segment elevating, $\Sigma$-ST) and myocardial ischemic scope (total point number of ST segment elevating, N-ST). At the end of the protocol, the animals sacrificed and heart taken out. Under the coronary occlusion spot, the ventricle was transversely divided into 5 pieces of equal thickness. The pieces were then infiltrated with N-BT staining solution at $25^{\circ} \mathrm{C}$ for $15 \mathrm{~min}$. Both the ischemic area (NBT non-stained area, white to grey) and non-ischemic area (N-BT-stained area, dark brown) were determined by histological methods.

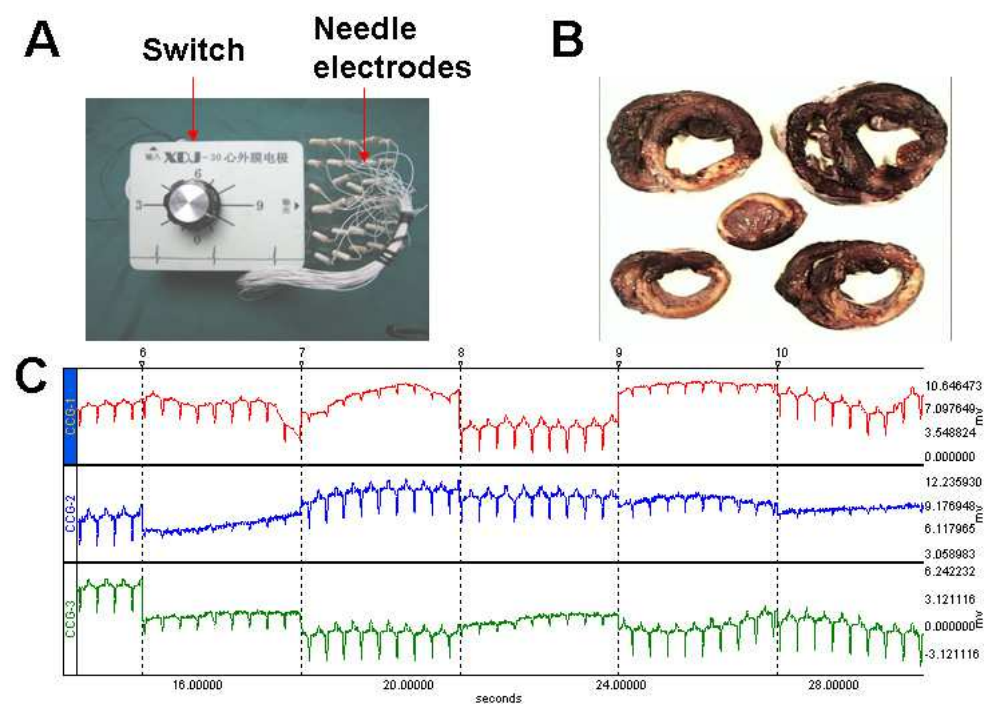

Fig. 6. BS-ECG machine (A), myocardium slices (B) (dark brown: live muscle; white: dead muscle), and BS-ECG (C).

\section{Conclusion}

The last few decades have seen significant advancement in the therapy of ischemic heart diseases. This is a direct outcome of the increasing knowledge of the molecular mechanisms involved during an ischemic insult of the myocardium. Another important factor is the development of animal model study, both academically and practically. The miniature swine was now widely used as research subject because of its anatomic similarity in coronary circulation to human beings (Hughes et al., 2003). Chest-closed improvement has been one of breakthroughs since birth of interventional technology. The newly introduced intervention technique avoided thoracotomy and disturbance to the environment of thoracic cavity. Moreover, cardiovascular variations could be chronically, continually and systematically observed in these models.

Despite tremendous advances in cardiovascular research and clinical therapy, ischemic heart disease remains the leading cause of serious morbidity and mortality in western society and is growing in developing countries. For researchers involving in basic science of 
heart diseases, one of the primary impediments to successful drug $R \& D$ is the frequent failure of successfully translating positive results obtained in animal models to human disease. To a large degree, this discrepancy is secondary to the substantial biological differences between species. We hope the information contained in this chapter will be helpful for researchers to consider prospectively their research plan regarding with myocardial ischemia/infarction animal study, which will someday bridge the gap between disease and the key molecular processes involved.

\section{Acknowledgment}

We thank Drs. Zhen Yu and Lei Li for their hard work with animal study. This work was supported by National Natural Science Foundation of China (30830118), National Key Scientific Program (2006BAI08B01-06, 2009ZX09303-003 and 2009ZX09502-017).

\section{References}

Baks, T., et al. (2006). Multislice computed tomography and magnetic resonance imaging for the assessment of reperfused acute myocardial infarction. J Am Coll Cardiol, Vol. 48, No. 1, pp.144-152

Bohl, S., et al. (2009). Advanced methods for quantification of infarct size in mice using three-dimensional high-field late gadolinium enhancement MRI. Am J Physiol Heart Circ Physiol, Vol. 296, No. 4, pp.H1200-1208

Bolukoglu, H., et al. (1992). An animal model of chronic coronary stenosis resulting in hibernating myocardium. Am J Physiol, Vol. 263, No. 1 Pt 2, pp.H20-29

Buecker, A., et al. (2005). A feasibility study of contrast enhancement of acute myocardial infarction in multislice computed tomography: comparison with magnetic resonance imaging and gross morphology in pigs. Invest Radiol, Vol. 40, No. 11, pp.700-704

Ch'en, F. F., et al. (1998). Modelling myocardial ischaemia and reperfusion. Prog Biophys Mol Biol, Vol. 69, No. 2-3, pp.515-538

Dogne, J. M., et al. (2005). Characterization of an original model of myocardial infarction provoked by coronary artery thrombosis induced by ferric chloride in pig. Thromb Res, Vol. 116, No. 5, pp.431-442

Elzinga, W. E. (1969). Ameroid constrictor: uniform closure rates and a calibration procedure. J Appl Physiol, Vol. 27, No. 3, pp.419-421

Falk, E., et al. (1995). Coronary plaque disruption. Circulation, Vol. 92, No. 3, pp.657-671

Fischell, T. A., et al. (2006). Real-time detection and alerting for acute ST-segment elevation myocardial ischemia using an implantable, high-fidelity, intracardiac electrogram monitoring system with long-range telemetry in an ambulatory porcine model. $J$ Am Coll Cardiol, Vol. 48, No. 11, pp.2306-2314

Fuchs, S., Kornowski, R. (2005). Correlation between endocardial voltage mapping and myocardial perfusion: implications for the assessment of myocardial ischemia. Coron Artery Dis, Vol. 16, No. 3, pp.163-167

Geurts, A. M., et al. (2009). Knockout rats via embryo microinjection of zinc-finger nucleases. Science, Vol. 325, No. 5939, pp.433 
Guth, B. D., et al. (1990). Myocardial lactate release during ischemia in swine. Relation to regional blood flow. Circulation, Vol. 81, No. 6, pp.1948-1958

Halvorsen, P. S., et al. (2009). Detection of myocardial ischaemia by epicardial accelerometers in the pig. Br J Anaesth, Vol. 102, No. 1, pp.29-37

Hennan, J. K., et al. (2001). Effects of selective cyclooxygenase-2 inhibition on vascular responses and thrombosis in canine coronary arteries. Circulation, Vol. 104, No. 7, pp.820-825

Higuchi, T., et al. (2007). Characterization of normal and infarcted rat myocardium using a combination of small-animal PET and clinical MRI. J Nucl Med, Vol. 48, No. 2, pp.288-294

Huang, Y., et al. (2004). Remodeling of the chronic severely failing ischemic sheep heart after coronary microembolization: functional, energetic, structural, and cellular responses. Am J Physiol Heart Circ Physiol, Vol. 286, No. 6, pp.H2141-2150

Hughes, G. C., et al. (2003). Translational physiology: porcine models of human coronary artery disease: implications for preclinical trials of therapeutic angiogenesis. J Appl Physiol, Vol. 94, No. 5, pp.1689-1701

Hutchison, S. J., et al. (2005). Dehydroepiandrosterone sulfate induces acute vasodilation of porcine coronary arteries in vitro and in vivo. J Cardiovasc Pharmacol, Vol. 46, No. 3 , pp.325-332

Inou, T., et al. (1980). A newly developed X-ray transparent ameroid constrictor for study on progression of gradual coronary stenosis. Basic Res Cardiol, Vol. 75, No. 4, pp.537543

Koning, M. M., et al. (1993). Intracoronary trimetazidine does not improve recovery of regional function in a porcine model of repeated ischemia. Cardiovasc Drugs Ther, Vol. 7, No. 5, pp.801-807

Kraitchman, D. L., et al. (2000). A minimally invasive method for creating coronary stenosis in a swine model for MRI and SPECT imaging. Invest Radiol, Vol. 35, No. 7, pp.445451

Krombach, G. A., et al. (2005). Minimally invasive close-chest method for creating reperfused or occlusive myocardial infarction in swine. Invest Radiol, Vol. 40, No. 1, pp.14-18

Laham, R. J., et al. (2000). Intrapericardial delivery of fibroblast growth factor-2 induces neovascularization in a porcine model of chronic myocardial ischemia. J Pharmacol Exp Ther, Vol. 292, No. 2, pp.795-802

Leadley, R. J., Jr., et al. (2000). Contribution of in vivo models of thrombosis to the discovery and development of novel antithrombotic agents. J Pharmacol Toxicol Methods, Vol. 43, No. 2, pp.101-116

Li, L. (1978). The improvement of epicardial electrogram methods. New Med Phar J, Vol. 1, No. 11, pp.52-53

Li, X. Z., et al. (2009). Establishment of coronary heart disease model of coronary atherosclerosis in mini-swines. Zhongguo Zhong Xi Yi Jie He Za Zhi, Vol. 29, No. 3, pp.228-232

Lichtig, C., et al. (1975). Basic fuchsin picric acid method to detect acute myocardial ischemia. An experimental study in swine. Arch Pathol, Vol. 99, No. 3, pp.158-161

Liu, J. X., et al. (2007a). Effects of Corocalm (shuguan capsule) on acute myocardial ischemia in anesthetized dogs. Chin J Integr Med, Vol. 13, No. 3, pp.206-210 
Liu, J. X., et al. (2002). Effects of recombinant staphylokinase on coronary thrombosis in Chinese experimental miniature swine. Acta Pharmacol Sin, Vol. 23, No. 6, pp.509515

Liu, J. X., et al. (2007b). Cardioprotective effects of diltiazem reevaluated by a novel myocardial ischemic model in Chinese miniature swine. Acta Pharmacol Sin, Vol. 28, No. 1, pp.52-57

Ludemann, L., et al. (2007). Usage of the T1 effect of an iron oxide contrast agent in an animal model to quantify myocardial blood flow by MRI. Eur J Radiol, Vol. 62, No. 2, pp.247-256

Mahnken, A. H., et al. (2005). Assessment of myocardial viability in reperfused acute myocardial infarction using 16-slice computed tomography in comparison to magnetic resonance imaging. J Am Coll Cardiol, Vol. 45, No. 12, pp.2042-2047

Moreno, C., et al. (2011). Creation and characterization of a Renin knockout rat. Hypertension, Vol. 57, No. 3, pp.614-619

Nash, M. P., et al. (2003). Imaging electrocardiographic dispersion of depolarization and repolarization during ischemia: simultaneous body surface and epicardial mapping. Circulation, Vol. 107, No. 17, pp.2257-2263

Naslund, U., et al. (1992). A closed-chest myocardial occlusion-reperfusion model in the pig: techniques, morbidity and mortality. Eur Heart J, Vol. 13, No. 9, pp.1282-1289

Odenstedt, J., et al. (2003). Endocardial electromechanical mapping in a porcine acute infarct and reperfusion model evaluating the extent of myocardial ischemia. I Invasive Cardiol, Vol. 15, No. 9, pp.497-501

Park, W. H., et al. (1985). Morphological changes in the coronary circulation following experimental myocardial ischemia in swine. Artery, Vol. 12, No. 5, pp.286-300

Rodriguez, B., et al. (2006). Modeling cardiac ischemia. Ann N Y Acad Sci, Vol. 1080, No. pp.395-414

Romson, J. L., et al. (1980). Electrical induction of coronary artery thrombosis in the ambulatory canine: a model for in vivo evaluation of anti-thrombotic agents. Thromb Res, Vol. 17, No. 6, pp.841-853

Seta, F., et al. (2009). Renal and cardiovascular characterization of COX-2 knockdown mice. Am J Physiol Regul Integr Comp Physiol, Vol. 296, No. 6, pp.R1751-1760

Skrzypiec-Spring, M., et al. (2007). Isolated heart perfusion according to Langendorff---still viable in the new millennium. J Pharmacol Toxicol Methods, Vol. 55, No. 2, pp.113126

Takahashi, M., et al. (2005). Effects of angiotensin I-converting enzyme inhibitor and angiotensin II type 1 receptor blocker on the right ventricular sarcoglycans and dystrophin after left coronary artery ligation. Eur J Pharmacol, Vol. 522, No. 1-3, pp.84-93

Tomita, S., et al. (2002). Improved heart function with myogenesis and angiogenesis after autologous porcine bone marrow stromal cell transplantation. J Thorac Cardiovasc Surg, Vol. 123, No. 6, pp.1132-1140

Wright, J., et al. (2009). Quantification of myocardial area at risk with T2-weighted CMR: comparison with contrast-enhanced CMR and coronary angiography. JACC Cardiovasc Imaging, Vol. 2, No. 7, pp.825-831

Yan, L., et al. (2006). Autophagy: a novel protective mechanism in chronic ischemia. Cell Cycle, Vol. 5, No. 11, pp.1175-1177 
Yan, L., et al. (2005). Autophagy in chronically ischemic myocardium. Proc Natl Acad Sci U S A, Vol. 102, No. 39, pp.13807-13812

$\mathrm{Yu}, \mathrm{Y}$. , et al. (2005). Differential impact of prostaglandin $\mathrm{H}$ synthase 1 knockdown on platelets and parturition. J Clin Invest, Vol. 115, No. 4, pp.986-995

$\mathrm{Yu}, \mathrm{Y}$., et al. (2006). Genetic model of selective COX2 inhibition reveals novel heterodimer signaling. Nat Med, Vol. 12, No. 6, pp.699-704

$\mathrm{Yu}, \mathrm{Y}$. , et al. (2007a). Targeted cyclooxygenase gene (ptgs) exchange reveals discriminant isoform functionality. J Biol Chem, Vol. 282, No. 2, pp.1498-1506

$\mathrm{Yu}, \mathrm{Z}$., et al. (2007b). Protective effects of Shuangshen Ningxin capsule on miniature swine after myocardial ischemia by intervention. Zhongguo Zhong Yao Za Zhi, Vol. 32, No. 16, pp.1695-1699 


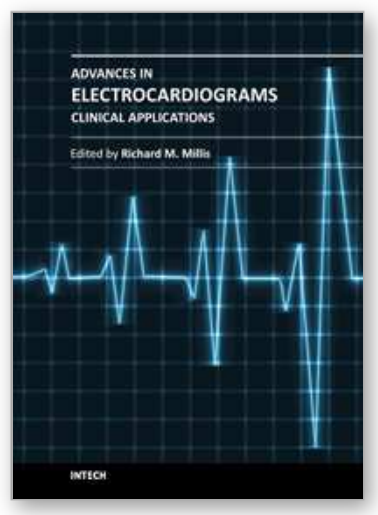

\author{
Advances in Electrocardiograms - Clinical Applications \\ Edited by PhD. Richard Millis
}

ISBN 978-953-307-902-8

Hard cover, 328 pages

Publisher InTech

Published online 25, January, 2012

Published in print edition January, 2012

Electrocardiograms have become one of the most important, and widely used medical tools for diagnosing diseases such as cardiac arrhythmias, conduction disorders, electrolyte imbalances, hypertension, coronary artery disease and myocardial infarction. This book reviews recent advancements in electrocardiography. The four sections of this volume, Cardiac Arrhythmias, Myocardial Infarction, Autonomic Dysregulation and Cardiotoxicology, provide comprehensive reviews of advancements in the clinical applications of electrocardiograms. This book is replete with diagrams, recordings, flow diagrams and algorithms which demonstrate the possible future direction for applying electrocardiography to evaluating the development and progression of cardiac diseases. The chapters in this book describe a number of unique features of electrocardiograms in adult and pediatric patient populations with predilections for cardiac arrhythmias and other electrical abnormalities associated with hypertension, coronary artery disease, myocardial infarction, sleep apnea syndromes, pericarditides, cardiomyopathies and cardiotoxicities, as well as innovative interpretations of electrocardiograms during exercise testing and electrical pacing.

\title{
How to reference
}

In order to correctly reference this scholarly work, feel free to copy and paste the following:

Jianxun Liu and Xinzhi Li (2012). Novel Porcine Models of Myocardial Ischemia/Infarction - Technical Progress, Modified Electrocardiograms Validating, and Future Application, Advances in Electrocardiograms Clinical Applications, PhD. Richard Millis (Ed.), ISBN: 978-953-307-902-8, InTech, Available from: http://www.intechopen.com/books/advances-in-electrocardiograms-clinical-applications/novel-porcine-modelsof-myocardial-ischemia-infarction-technical-progress-modified-electrocardiogram

\section{INTECH}

open science | open minds

\section{InTech Europe}

University Campus STeP Ri

Slavka Krautzeka 83/A

51000 Rijeka, Croatia

Phone: +385 (51) 770447

Fax: +385 (51) 686166

www.intechopen.com

\section{InTech China}

Unit 405, Office Block, Hotel Equatorial Shanghai

No.65, Yan An Road (West), Shanghai, 200040, China

中国上海市延安西路65号上海国际贵都大饭店办公楼 405 单元

Phone: +86-21-62489820

Fax: +86-21-62489821 
(C) 2012 The Author(s). Licensee IntechOpen. This is an open access article distributed under the terms of the Creative Commons Attribution 3.0 License, which permits unrestricted use, distribution, and reproduction in any medium, provided the original work is properly cited. 